\title{
Urticaria multiforme: A commonly misdiagnosed entity
}

\section{Reena K Sharma, Mudita Gupta, Kuldeep Verma, Saru Thakur}

Department of Dermatology, Venereology and Leprosy, Indira Gandhi Medical College, Shimla, Himachal Pradesh, India

Corresponding author: Dr. Mudita Gupta, E-mail: muditadrgupta@yahoo.com

\begin{abstract}
Cutaneous hypersensitivity reactions in paediatric age group have varied presentations. Urticaria multiforme is a characteristic morphologic type of self resoluting hypersensitivity reaction secondary to drugs or infections, characterised by acute onset annular, polycyclic wheals with ecchymotic centres. We present an eighteen month female and a 5 years male child who presented to us with itchy erythematous blanching annular wheals with dusky centre and were diagnosed as urticaria multiforme. It resolves completely with antihistamines and therefore need to be differentiated from severe reaction patterns.
\end{abstract}

Key words: Urticaria multiforme; Hypersensitivity; Dusky centre

\section{INTRODUCTION}

Urticaria multiforme (UM) is also known as acute annular urticaria. It is characterized by annular, arciform, polycyclic erythematous blanchable wheals with violaceus dusky centre [1]. These lesions are sudden in onset and subside in an hour or two, otherwise resembling erythema multiforme. There is associated acral edema [1]. We report two cases of urticaria multiforme to emphasize that UM is a distinct variant of urticaria and should be differentiated from other disorders with similar morphology.

\section{CASE REPORT}

\section{Case 1}

An eighteen months female child presented with history of fever and cough for 5 days and itchy erythematous lesions all over the body for past 2 days. The lesions were persisting for 24 hours with onset of new lesions at other locations. The child was otherwise active. On examination child was febrile with a temperature of $100.4^{0} \mathrm{~F}$. There was no lymphadenopathy or joint swelling. Cutaneous examination showed multiple wheals which were erythematous annular and polycyclic, present all over the body with dusky purpuric centre (Fig. la) and associated edema of hands and feet (Fig. lb). Dermographism was positive. Haematological examination, urine microscopy, erythrocyte sedimentation rate were within normal limits. Antstreptolysin O, blood and urine culture were non-contributory. C-reactive protein was slightly raised. As the clinical presentation of patient was quite characteristic she was diagnosed to be having urticaria multiforme and prescribed antihistamines; the wheals disappeared within few hours but few new lesions appeared at different location. Antihistamines were continued and the child was completely asymptomatic after 2 weeks follow up, with no pigmentation or scarring.

\section{Case 2}

A 5 years male child with history of discharge from left ear for 4 days, for which he was taking amoxicillin presented with severely itchy erythematous raised plaques all over the body for 2 days. On examination child was afebrile, but irritable due to pruritus. Cutaneous examination showed multiple variable sized erythematous annular wheals present all over 
the body (Figs. 2a and 2b) and positive (Fig. 2c). All routine investigations were normal. Child was given antihistamines; lesions subsided within 24 hours of medication but reappeared next day. The dose of antihistamine was doubled and child was completely asymptomatic after 2 weeks (Fig. 2d).

\section{DISCUSSION}

In 1997 Tamayo-Sánchez et al observed acute onset of annular urticarial and named the entity as acute annular urticarial(AAU) [2]. Ten years later Shah et al described acute urticarial lesions with dusky centre and due to its similarity with erythema multiforme, named it as urticaria multiforme(UM) [3]. Both these studies had similar diagnostic criteria except for few differences. The latter studies considered UM to be a variant of acute urticaria with dermographism [4]. Both the terms AAU and UM are now used synonymously. Terminology UM is debatable because of the absence of variable forms and the nomenclature of erythema multiforme like urticaria was proposed [1].

Urticaria multiforme (UM) is a morphological variant of acute urticaria. It is seen most commonly in children older than 4 months but less than 4 years of age [5] Occasionally UM in neonates and adolescents have also been reported [1]. UM is a hypersensitivity reaction usually secondary to viral infections or certain drugs. Patient usually has a history of fever, sore throat, otitis media, upper respiratory, gastrointestinal infection preceding the episode. There may be history of onset of lesions after intake of drugs like amoxicillin, cephalosporins or macrolides, furazolidones, aspirin or after immunization [6]. Like any urticaria, the lesions start as erythematous macule, papule which progress to itchy, blanchable wheals which are transient ( $<24$ hours), healing without pigmentation or scarring. Morphology of the lesion in erythema multiforme (EM) and UM is similar with annular, polycyclic lesions and dusky centre. In contrast to target lesions in EM lesions, they are targetoid in UM. The lesions in EM persist for few days while they are transient in UM. Also $\mathrm{UM}$ is associated with dermographism, pruritus and acral edema which are absent in EM. Other cutaneous hypersensitivity responses with similar presentation are urticarial vasculitis and serum sickness like illness [7]. Urticarial vasculitis(UV) lesion is annular but not targetoid, persisting for more than 24 hours, burning is the common complaint rather than pruritus. UV is uncommon in paediatric age group less than 5 years. UV unlike UM heals with hyperpigmentation. In

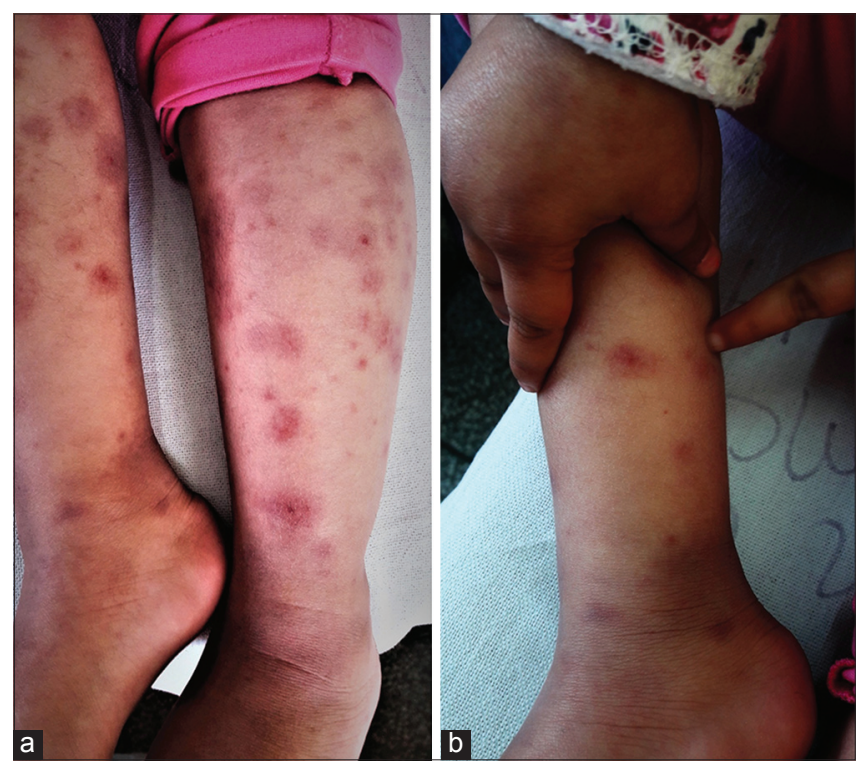

Figure 1: (a) Erythematous, targetoid wheals on both lower limbs (b) Acral edema with targetoid lesions.
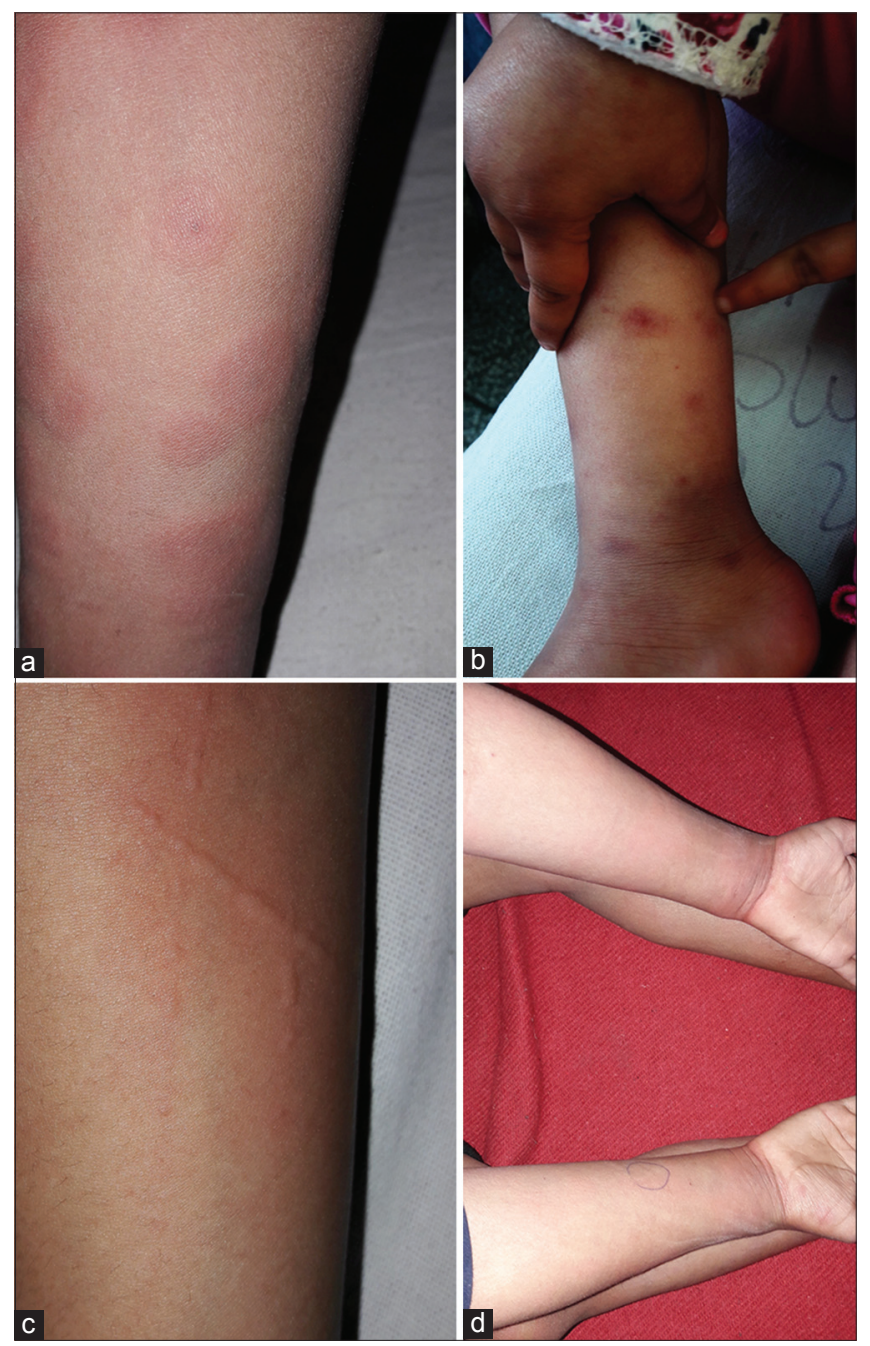

Figure 2: (a) Erythematous wheals with dusky purpuric centre over forearm, (b) Lesions showing targetoid morphology over bilateral upper and lower limbs (c) Remission of lesion with medication within 24 hours (d) Dermographism after stroking forearm of child. 
serum sickness like reaction child is sick with fever, lymphadenopathy, arthralgia and acral oedema. Acute haemorrhagic edema of infancy may also present as a targetoid lesion but is purpuric and leaves hyperpigmentation [8].

Clinical history and examination are sufficient to diagnose UM. Histopathology of the lesions show dermal edema and perivascular lymphocytic with eosinophilic infiltrate, with a normal epidermis in contrast to basal layer necrosis and interface dermatitis seen in EM and no inflammatory infiltrate in urticaria. Routine haematological tests, C-reactive proteins, IgE levels are normal.

UM being a hypersensitivity response is not an uncommon disease in children and is self limiting in 10-14 days [9]. It needs to be clinically differentiated from other similar but severe reaction pattern, so that the load of investigations can be minimised. Antihistamines are the only treatment required.

\section{CONSENT}

The examination of the patient was conducted according to the Declaration of Helsinki principles.

\section{REFERENCES}

1. Tarnes L, Patel T, Skinner RB. Urticaria multiforme - a case report. Pediatr Dermatol. 2011;28:436-8.

2. Tamayo-Sanchez L, Ruiz-Maldonado R, Laterza A. Acute annular urticaria in infants and children. Pediatr Dermatol. 1997;14:231-4.

3. Shah KN, Honig PJ, Yan AC. "Urticaria multiforme": a case series and review of acute annular urticarial hypersensitivity syndromes in children. Pediatrics. 2007;119:e1177-e83.

4. Sempau L, Martín-Sáez E, Gutiérrez-Rodríguez C, GutiérrezOrtega MC. Urticaria Multiforme: A Report of 5 Cases and a Review of the Literature. Actas Dermosifiliogr. 2016;107:e 1-5.

5. Guerrier G, Daronat J-M, Deltour R. Unusual Presentation of Acute Annular Urticaria: A Case Report. Case Rep Dermatol Med. 2011;2011:604390.

6. Fung IN, Berger EM, Castelo-Soccio L, Brown Whitehorn TF. Urticaria multiforme in an 18-year-old girl. Allergy Clin Immunol Pract. 2013:1;520-1.

7. Mathur AN, Mathes EF. Urticaria mimickers in children. Dermatol Ther. 2013:26; 467-75.

8. Emer JJ, Bernardo SG, Kovalerchik O, Moneeb Ahmad BS. Clin Aesthet Dermatol. 2013:6;34-9.

9. Myers SR, Lavelle J. Picture of the month—quiz case. Pneumonia with associated urticaria multiforme rash. Arch Pediatr Adolesc Med. 2009:163;1157.

Copyright by Reena K Sharma, et al. This is an open access article distributed under the terms of the Creative Commons Attribution License, which permits unrestricted use, distribution, and reproduction in any medium, provided the original author and source are credited.

Source of Support: Nil, Conflict of Interest: None declared. 